\title{
On the wing
}

\author{
I flygande fart
}

\author{
Jonas Waldenström ${ }^{1}$ (iD \& Martin Stervander ${ }^{2}$ \\ 1'Department of Biology and Environmental Science, Linnaeus University, 39182 Kalmar, Sweden | jonas.waldenstrom@ \\ Inu.se ${ }^{2}$ Bird Group, Natural History Museum, Akeman St, Tring, Hertfordshire HP23 6AP, UK | martin@stervander.com
}

BIRDS ARE FASCINATING. For one thing, they can do what we cannot, like flying-as easily as anything at that, like a flock of Common Swifts Apus apus hastening through the late summer skies with their piercing screams, or a Wandering Albatross Diomedea exulans whose tip of the wing slices the ocean surface while the bird rides the wave troughs. For another, their appearance, their song, and their behaviours are so incredibly variable. In short, if you have ever really seen a bird, you will be forever smitten.

This is certainly true for the authors of Ornis Svecica. Last year, we published papers on birds from the northernmost Sweden (Ferry et al. 2021) to Tristan da Cunha in the middle of the South Atlantic (Bond \& McClelland 2021), with a taxonomic breadth from Red-throated Loons Gavia stellata (Olsson et al. 2021) to Bramblings Fringilla montifringilla (Svensson 2021), and on subjects ranging from conservation biology to counting techniques. This is one of the exciting aspects of being a journal editor-one never knows what manuscripts are coming and which discoveries our authors have made. The breadth is also apparent in the type of article; in 2021 we published 150 pages, comprising seven research papers, two short communications, one review paper, four book reviews, a forum piece, and an obituary. One thing that particularly characterizes Ornis Svecica is that we publish many works by amateur ornithologists. This is an important niche that we aim to continue to fill.

This year's volume is the $32^{\text {nd }}$ since the journal was established and the third with entirely digital publishing. The change has resulted in a simplified distribution-especially to readers outside Sweden, who might otherwise have overlooked our papers. We regard the distribution and outreach as a long-term effort, and although there are things that need adjusting, a lot also works well. Last year saw a strong increase in the number of visits to the journal website, with no less than 27,986 viewings of abstracts and 16,855 downloads of pdf files!

Among last year's contributions, a paper by Reino Andersson on the covariation between systematic counts and spontaneously reported figures of staging waders (Andersson 2021) was the top read, with 789 views. The same author's contributions from previous 
years about the plumage variation (Andersson 2019a) and population development (Andersson 2019b) of the European Stonechat Saxicola rubicola top the list of most read papers last year, with 911 and 849 views respectively. We hope that you, as a reader of Ornis Svecica, will help spread our papers in your own networks and channels. After all, the journal is free to publish in and free to read-quite the rarity in the journal world!

For the new year, we extend our editorial board with three members (Figure 1):

MURIELLE ALUND is an evolutionary researcher at

Uppsala University who studies Pied Flycatchers

Ficedula hypoleuca on Öland and Three-spined

Sticklebacks Gasterosteus aculeatus in Iceland.

GUNNAR GUNNARSSON is an animal ecologist and assistant professor at Kristianstad University, with wetland birds as his specialty.

FREDRIK ANDREASSON is an evolutionary ecologist at

Lund University, focusing on the thermophysiology of tits (Paridae).

For further presentations of the new editors, see the journal's website. We would also like to thank Helena Westerdahl, who has left the editorial board after a couple of years.

This year's first two papers can be said to reflect the soul of Ornis Svecica. The first is penned by Reino Andersson, who has systematically mapped the entrance of another winner of our changing avifauna. The bird in question is the White-spotted Bluethroat Luscinia svecica cyanecula, a vocal equilibrist and visual gem that colonized south-western Sweden over the past few years. It prefers habitats that are rather abundant-such as reedbeds, wet trenches, and vegetated shores-and we may therefore expect continued increase over the coming years (Andersson 2022). The cover of Volume 32 features one of the White-spotted Bluethroat males that Reino writes about, in a photo taken by Anna Högberg at Hjälms våtmark, Halland, 15 July 2018.

Conversely, the second paper-which is written by Carl-Gustav Thulin, Malin Sörhammar, and Jonas Bohlin-centres on a species that has gone extinct from Sweden, the Black Stork Ciconia nigra. This iconic bird has very specific requirements for their breeding environment, including relatively undisturbed forest with adjacent wetlands, the occurrence of which has been reduced by modern forestry over the last century. In their paper, Thulin et al. (2022) use species distribution models to identify areas that would be suitable to Black Storks today, and which might be the targets of a Swedish reintroduction programme. They note that it is exactly 100 years since the reintroduction of another locally extinct species to Sweden, the European beaver Castor fiber. Albeit not a bird, the habitat requirements of this rodent largely overlaps with those of the Black Stork (Thulin et al. 2022).

At this point, we do not want to reveal too much, but several exciting papers are in the pipeline. You are most welcome to contact the editors if you might have a contribution to Ornis Svecica.
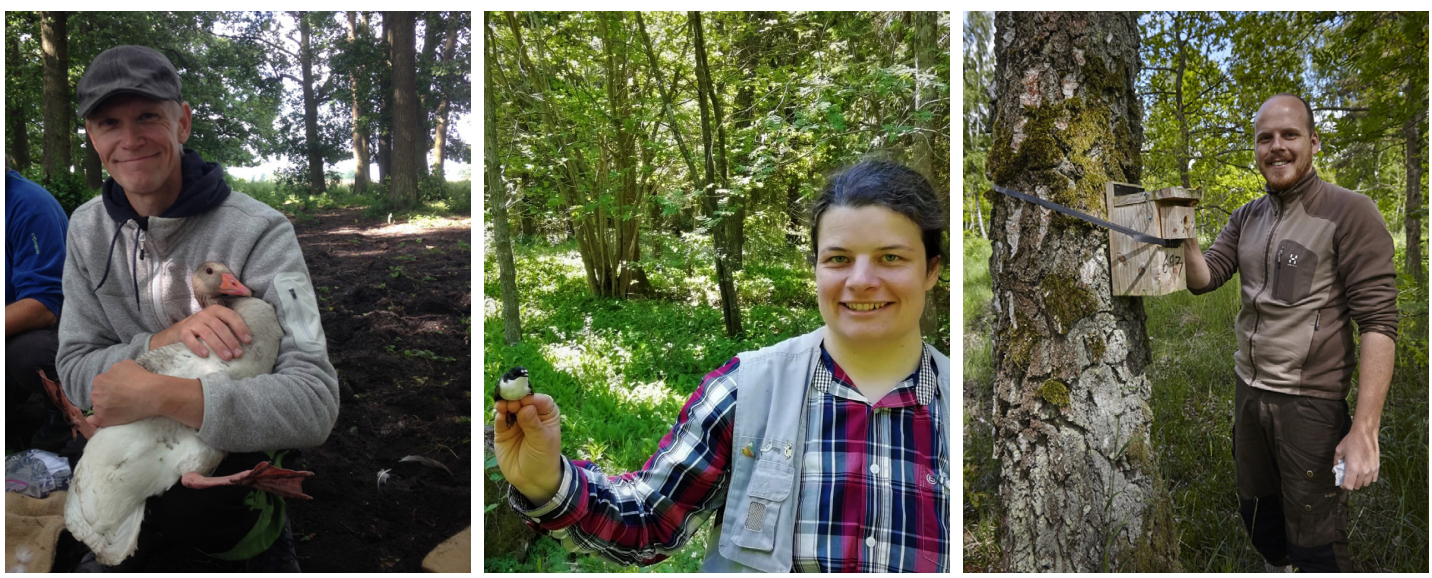

FIGURE 1. Ornis Svecica's three new editors during field work: Gunnar Gunnarsson, Murielle Ålund, and Fredrik Andreasson. - Ornis Svecicas tre nya redaktörer under fältarbete: Gunnar Gunnarsson, Murielle Ålund och Fredrik Andreasson. 


\section{References}

Andersson R. 2019a. Plumage variation of males in Scandinavian populations of European Stonechat Saxicola rubicola. Ornis Svecica 29: 76-83. https://doi.org/10.34080/os.v29.19691

Andersson R. 2019b. Population development of European Stonechat Saxicola rubicola during the initial phase of its expansion in Western Sweden. Ornis Svecica 29: 26-36. https://doi.org/10.34080/ os.v29.19555

Andersson R. 2021. Covariation between systematic counts and spontaneous reporting of staging waders at the Getterön Nature Reserve, southwestern Sweden. Ornis Svecica 31: 34-43. https://doi. org/10.34080/os.v31.22270

Andersson R. 2022. Immigration process of White-spotted Bluethroat Luscinia svecica cyanecula at the Swedish west coast. Ornis Svecica 32: 5-13. https://doi.org/10.34080/os.v32.23429

Bond AL \& McClelland G. 2021. Diet of the introduced Gough Moorhen Gallinula comeri on Tristan da Cunha. Ornis Svecica 31: 107-112. https://doi.org/10.34080/os.v31.23476
Ferry, B., Ekenstedt, J., \& Green, M. 2021. Densities of the Eurasian Threetoed Woodpecker Picoides tridactylus calculated from sap row surveys are on par with estimates from fixed route bird censusing. Ornis Svecica 31: 94-106. https://doi.org/10.34080/os.v31.22416

Olsson, C., Pettersson, J., \& Johansson, F. 2021. Breeding biology of the Red-throated Loon Gavia stellata on the Holmöarna archipelago, Northern Sweden. Ornis Svecica 31: 1-18. https://doi. org/10.34080/os.v31.22276

Svensson, T. 2021. A review of mass concentrations of Bramblings Fringilla montifringilla: implications for assessment of large numbers of birds. Ornis Svecica 31: 44-67. https://doi.org/10.34080/ os.v31.22031

Thulin C-G, Sörhammar M \& Bohlin J. 2022. Black Stork Back: Species distribution model predictions of potential habitats for Black Stork Ciconia nigra in Sweden. Ornis Svecica 32: 14-25. https://doi. org/10.34080/os.v32.22081

\section{Svensk version}

Det är lätt att fascineras av fåglar. Dels för att de kan göra sådant som vi inte kan, som att flyga - därtill med en lekande lätthet, som när en sensommarflock med tornseglare Apus apus drar förbi i ilande, skriande flykt, eller när vandringsalbatrossen Diomedea exulans rider i vågdalarna och med det yttersta av vingspetsen skär ett litet streck i vattenytan - dels för att de så variabla i utseende, beteenden och sång. Kort och gott: den som en gång verkligen sett en fagel är för alltid biten.

Detta gäller sannerligen författarna i Ornis Svecica. Under föregående år publicerade vi uppsatser om fåglar från nordligaste Sverige (Ferry m. fl. 2021) till Tristan da Cunha i mitten av Sydatlanten (Bond \& McClelland 2021), med en taxonomisk bredd från smålommar $G a$ via stellata (Olsson m. fl. 2021) till bergfinkar Fringilla montifringilla (Svensson 2021), och ämnen som sträcker sig från bevarandebiologi till beteende. Det är en av de spännande sakerna med att vara redaktör för en tidskrift, att man aldrig riktigt vet vilket manus som är på ingång och vilka upptäckter som våra författare har gjort. Bredden utmärker sig även i typen av texter; under 2021 publicerade vi 150 sidor, vilka omfattade sju uppsatser, två korta rapporter, en översiktsartikel, fyra bokrecensioner, en forumtext och en minnesruna. Något som utmärker Ornis Svecica är att vi i hög grad publicerar arbeten gjorda av amatörornitologer. Det är en viktig nisch som vi ämnar fortsätta att fylla.
Årets årgång är den 32:a sedan starten och den tredje med helt digital publicering. Förändringen har medfört en enklare distribution, särskilt till våra läsare utanför Sveriges gränser som annars kanske missat uppsatserna. Vi ser det som ett långsiktigt arbete och det finns en del saker att slipa på, men mycket fungerar väl. Förra året såg en kraftig ökning av besöken på tidskriftens webbplats, med inte mindre än 27986 visningar av sammanfattningar (abstract) och 16855 nedladdningar av uppsatser som pdf-filer!

Bland förra årets texter toppas läslistan Reino Anderssons uppsats om samvariationen mellan systematiska inventeringar av vadare och spontant rapporterade observationer (789 visningar; Andersson 2021) och samma författares alster från föregående år om svarthakade buskskvättans Saxicola rubicola dräktvariation (911 visningar; Andersson 2019a) respektive populationsutveckling (849 visningar; Andersson 2019b) toppar årets lista av mest lästa alster. Vi hoppas att ni läsare till Ornis Svecica vill vara delaktiga i att sprida våra uppsatser i de kanaler som ni använder. Tidskriften är ju gratis att publicera i och gratis att läsa - något av en raritet!

Det nya året ser också en delvis ny redaktion, med tre nya redaktörer (figur 1): Murielle Ålund är evolutionsforskare vid Uppsala universitet och studerar öländska svartvita flugsnappare Ficedula hypoleuca 
och isländska spiggar Gasterosteus aculeatus; Gunnar Gunnarsson är djurekolog och biträdande professor vid Högskolan Kristianstad, med våtmarksfåglar som sin specialitet; och Fredrik Andreasson är evolutionsekolog och adjunkt vid Lunds universitet, med fokus på mesars termofysiologi. En närmare presentation av de nya redaktörerna finns på tidskriftens webbplats. Vi vill också tacka Helena Westerdahl som lämnat redaktörsposten efter ett par år.

Årets första uppsatser speglar tidskriftens själ och hjärta på ett bra sätt. Den första är skriven av Reino Andersson, som tar sig an ämnet om en av den nya tidens vinnare $\mathrm{i}$ vår föränderliga fågelfauna. Fågeln $\mathrm{i}$ fråga är vitstjärnig blåhake Luscinia svecica cyanecula, en skönsjungade juvel som de senaste åren börjat kolonisera sydvästligaste Sverige. Biotoperna den föredrar är ganska vanliga - som vassbälten, blöta diken och stränder med vegetation - och man kan förvänta sig en ökning under kommande år (Andersson 2022). Årgång 32 pryds av en av de vitstjärniga blåhakshannar som Reino skriver om, i ett foto taget av Anna Högberg vid Hjälms våtmark i Halland 15 juli 2018.
Årets andra uppsats är skriven av Carl-Gustav Thulin, Malin Sörhammar och Jonas Bohlin och behandlar en art som har försvunnit ur fågelfaunan, nämligen svart stork Ciconia nigra. Denna karaktäristiska fågel har högt ställda krav på sin häckningsmiljö, och vill ha relativt ostörd skog med riklig förekomst av våtmarker, något som det moderna skogsbruket inverkat på under de senaste hundra åren. I deras uppsats använder Thulin m. fl. (2022) artutbredningsmodeller för att identifiera områden som skulle passa för svart stork idag, och som skulle kunna ligga till grund för ett återplanteringsprojekt. Författarna noterar också att det $\mathrm{i}$ år är hundraårsjubileum för återintroduktionen av en tidigare utrotad art i Sverige, nämligen bävern Fiber castor. Denna speciella gnagare har liknande krav på sin livsmiljö som den svarta storken, och den rikliga bäverstammen kan således ses som ett lovande tecken för storkarna (Thulin m. fl. 2022).

Mer än så vill vi inte avslöja - flera spännande uppsatser är på gång. Varmt välkomna att kontakta redaktionen om du själv vill bidra med ett arbete.

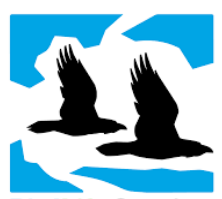

Birdlife Sverige

Ornis Svecica (ISSN 2003-2633) is an open access, peer-reviewed scientific journal published in English and Swedish by BirdLife Sweden. It covers all aspects of ornithology, and welcomes contributions from scientists as well as non-professional ornithologists. Accepted articles are published at no charge to the authors. Read papers or make a submission at os.birdlife.se.

Ornis Svecica (ISSN 2003-2633) är en fritt tillgänglig granskad vetenskaplig tidskrift som ges ut på svenska och engelska av BirdLife Sverige. Den täcker ornitologins alla områden och välkomnar bidrag från såväl forskare som icke-professionella ornitologer. Accepterade uppsatser publiceras utan kostnad för författarna. Läs uppsatser eller skicka in ditt bidrag på os.birdlife.se. 